\title{
SCHIZOPHRENIA: KRAEPELIN, SCHNEIDER AND BLEULER REVISITED
}

\section{INTRODUCTION}

The diagnosis of Schizophrenia is difficult because it is made from subjective experiences and there is no reliable biological marker or diagnostic test. For this reasons there is a maintained interest throughout time in refining the concept of the schizophrenic syndrome.

\section{DISCUSSION}

Kraepelin integrated various clinical pictures into a single nosological entity - "Dementia Praecox"- an illness that tended to

Magalhães M. ${ }^{1}$, Afonso P. ${ }^{1}$, Penedos S. ${ }^{1}$, Moreno L. ${ }^{1}$, Franco M. ${ }^{1}$, Ribeiro N. ${ }^{1}$, Fonseca I.

${ }^{1}$, Freire M. ${ }^{1}$, Nogueira J. ${ }^{1}$, Vieira J. ${ }^{1}$, Mendes R. ${ }^{1}$, Gamito A. ${ }^{1}$

${ }_{1}^{1}$ Departamento de Psiquiatria e Saúde Mental | Centro Hospitalar de Setúbal

begin at an early age ('praecox'), consisting of a series of states, being the common characteristic a peculiar destruction of the internal connections of the psychic personality. It had a chronic course with severe cognitive, behavioral and social impairment ('dementia'). For Kraepelin the two key features of this disorder are what we now refer to as negative symptoms and fragmenting of thought. He based his proposal in his longitudinal observations of a large number of clinical cases.

Bleuler, stated that early onset and progressive deterioration to dementia weren't exclusive to this disease, and so in 1908 he 1900 s coined the term Schizophrenia - which means fragmentation of the mind. Influenced by psychoanalysis he postulated a continuum ranging from schizophrenic psychosis to schizoid personality and schizothymic character.

$\mathrm{He}$ also introduced the concept of basic and accessory symptoms of Schizophrenia. Delusions and hallucinations, were considered as accessory, because they could occur in other conditions. Basic symptoms - the "four A's" = included Association disturbances, Ambivalence, Affective incongruence, and Autism. According to Bleuler, it was the presence of basic symptoms that gave schizophrenia its distinctive profile. Bleuler's diagnostic approach allowed for an expansion of the concept of Schizophrenia.

Schneider, based on clinical experience and the work of Karl Jaspers, sharpened the Schizophrenia diagnosis by discriminating between First and Second Rank symptoms - Table 1. The presence of one or more of the first rank symptoms were sufficient for the diagnosis. However, only a several second-rank symptoms would permit the diagnosis. Firstrank symptoms don't include negative symptoms nor any course-related criteria, which allowed for the diagnosis to become more restricted to psychotic illness and, consequentially, more narrow in scope and reliable.

Schneiders' symptoms were incorporated into the the Present State Examination, the first major structured interview developed for use in the International Pilot Study of Schizophrenia, and thereafter introduced into other standard diagnostic instruments. Since first-rank symptoms aren't always present and don't predict outcome, they were removed from the diagnostic criteria in DSM- 5, and deemphasized from ICD-11.

\section{Table 1 - Schneider's First-rank symptoms}

\begin{tabular}{|c|c|}
\hline Audible thoughts & Thought withdrawal or insertion \\
\hline Third-person hallucinations & Thought broadcasting \\
\hline Auditory hallucinations in the form of a 'running commentary' & Delusional perception \\
\hline Somatic (bodily, tactile) hallucinations & $\begin{array}{c}\text { Feelings or actions experienced as made or influenced by } \\
\text { external agents (passivity) }\end{array}$ \\
\hline
\end{tabular}

By being one of the most stigmatized mental condition, the question whether the word Schizophrenia should be abolished is already a matter of debate in many countries. Japan was the first country to adopt the new name of "TogoShitcho-Sho", which means Integration Disorder. Other countries in South East Asia have also followed the same path. It's very important to have more studies to assess the effects on stigma concerning the abolishment of the term Schizophrenia.

\section{CONCLUSION}

In the past century the concept of Schizophrenia has evolved, since Kreapelin, Bleuler and Schneider to the current classificatory systems. As of today it refers to a syndrome that can be diagnosed more reliably, which is essential for rational clinical practice.

\section{BIBLIOGRAPHY}

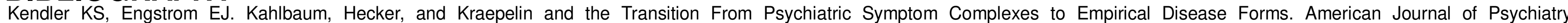

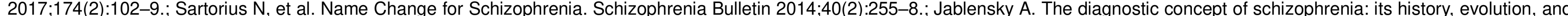

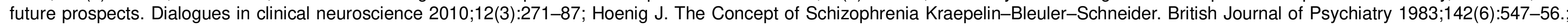

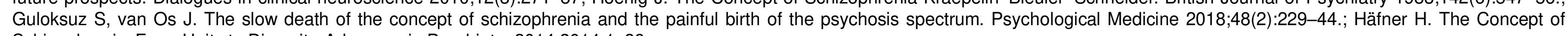
Schizophrenia: From Unity to Diversity. Advances in Psychiatry 2014;2014:1-39. 